\title{
SOLVABLE LIE ALGEBRAS OF DERIVATIONS OF POLYNOMIAL RINGS IN THREE VARIABLES
}

Let $\mathrm{K}$ be an algebraically closed field of characteristic zero, $\mathrm{A}=\mathrm{K}\left[\mathrm{x}_{1}, \mathrm{x}_{2}, \mathrm{x}_{3}\right]$ the polynomial ring in thre variables and $\mathrm{R}=\mathrm{K}\left(\mathrm{x}_{1}, \mathrm{x}_{2}, \mathrm{X}_{3}\right)$ the field of rational functions. If $L$ is a subalgebra of the Lie algebra $W_{3}(K)$ of all $K$-derivations of $A$, then $R L$ is a Lie algebra over $K$ and $\operatorname{dim}_{R} R L$ will be called the rank of $L$ over $R$. We study solvable subalgebras $L$ of $W_{3}(K)$ of rank 3 over $R$. It is proved that $\mathrm{L}$ is isomorphic to a subalgebra of the general affine Lie algebra af $_{3}(K)$ if $L$ contains an abelian ideal I of rank 3 over $R$. If $L$ has an ideal I with $r k_{R} I=2$, then $L$ is contained in a subalgebra $\bar{L}$ of $W / g(K)=\operatorname{Der}_{K} R$ such that $\bar{L}$ is an extension of a subalgebra of $\operatorname{aff}_{2}(F)$ by a subalgebra of dimension $\leq 2$, where $F$ is the field of constants of $I$ in $R$.

Introduction. Let $\mathrm{K}$ be an algebraically closed field of characteristic zero, $A=K\left[x_{1}, x_{2}, x_{3}\right]$ the polynomial ring in three variables and $R=K\left(x_{1}, x_{2}, x_{3}\right)$ the field of rational functions. Recall that a $K$-linear operator $D: A \rightarrow A$ is called a $K$-derivation on $A$ if $D$ satisfies the Leibniz's rule: $D(f g)=D(f) g+f D(g)$ for all $f, g \in A$. The Lie algebra $W_{3}(K)$ of all $K-$ derivations on $A$ is a very interesting mathematical object closely connected with groups of symmetries of partial differential equations. In case $\mathrm{K}$ is the field of real or comlpex numbers, all finite dimensional subalgebras of $W_{1}(K)$ and $W_{2}(K)$ were described in works of S. Lie, P. Olver, N. Kamran. The natural problem of classification of all finite dimensional subalgebras of $W_{3}(K)$ remains still open. S. Lie [7] began to study such subalgebras, but his classification even of nilpotent subalgebras is incomplete. U. Amaldi [1, 2] continued study of subalgebras of $W_{3}(K)$ but his classification is unsatisfactory. Note that the problem of classifying even nilpotent finite-dimensional subalgebras of $\mathrm{W}_{4}(\mathrm{~K})$ is wild (i.e. it contains a hopeless problem of classifying pairs of square matrices up to simultaneous similarity [3]).

We study finite dimensional solvable subalgebras of rank 3 over $R$ of the Lie algebra $W_{3}(K)$ (nilpotent subalgebras of $W_{3}(K)$ were studied in [10]). The main results of the paper: it is proved in Theorem 1 that a solvable finite dimensional subalgebra $L$ of $W_{3}(K)$ possessing an abelian ideal of rank 3 over $R$ is isomorphic to a subalgebra of the general affine Lie algebra aff $_{3}(K)$. If $L$ has an abelian ideal $I$ of rank 2 over $R$, then $L$ can be embedded in a subalgebra $\bar{L}$ of $\mathbb{H}_{3}(K)=\operatorname{Der}_{K} R$ such that $\bar{L}$ is an extension of a subalgebra of $\operatorname{aff}_{2}(\mathrm{~F})$ by a subalgebra of dimension $\leq 2$, where $F$ is the field of constants for the ideal $\mathrm{I}$ in the field $\mathrm{R}$.

Notations in the paper are standard. The ground field $\mathrm{K}$ is algebraically closed of characteristic zero. If $L$ is a subalgebra of the Lie algebra $W_{3}(K)$, then $F=F(L)$ is the field on constants of $L$ in $R=K\left(x_{1}, x_{2}, x_{3}\right)$ (we consider any derivation $D \in W_{3}(K)$ as derivation of $R$ in the natural way:

ISSN 1810-3022. Прикл. проблеми мех. і мат. - 2018. - Вип. 16. - С. 7- 13. 
$\left.D(f / g)=(D(f) g-f D(g)) / g^{2}\right)$. If $V$ is an $n$-dimensional vector space over $K$ and $g l(V)$ the Lie algebra of all linear operators on $V$ we can consider the semidirect product $\mathrm{gl}(\mathrm{V}) \cdot \mathrm{V}$, where $\mathrm{V}$ is considered as an abelian Lie algebra. The Lie algebra $\mathrm{gl}(\mathrm{V})$. $\mathrm{V}$ will be called the general affine Lie algebra and denoted by af $_{n}(K)$ (in case $K=R$ the Lie algebra aff $f_{n}(R)$ corresponds to the general affine Lie group $\mathrm{GA}_{n}(R)$ ).

Subalgebras with an abelian ideal of rank 3 over $R$.

The next two lemmas contain standard facts about derivations (see for example, [8]). More information about derivations of polynomial rings can be found in [9].

Lemma 1 Let $D_{1}, D_{2} \in W_{3}(K)$ and $a, b \in R$. Then $\left[a D_{1}, b D_{2}\right]=a b\left[D_{1}, D_{2}\right]+a D_{1}(b) D_{2}-b D_{2}(a) D_{1}$. If $\left[\mathrm{D}_{1}, \mathrm{D}_{2}\right]=0$, then $\left[a \mathrm{D}_{1}, b \mathrm{D}_{2}\right]=\mathrm{aD}_{1}(\mathrm{~b}) \mathrm{D}_{2}-\mathrm{bD}_{2}(\mathrm{a}) \mathrm{D}_{1}$.

Lemma 2 If $L \subseteq W_{3}(K)$ and $F=F(L)$ the field of constants for $L$ in $R$, then $F L$ is a Lie algebra over $F$. If $L$ is abelian, nilpotent or solvable then so is $F L$.

Lemma 3 Let $D_{1}, K, D_{n}$ be a basis of the vector space $H_{3}(K)$ over the field $R$. Then $\left.\right|_{i=1} ^{n} K_{e r D}=K$.

Proof. Suppose that $\mid{ }_{i=1}^{n} K \operatorname{Ker} D_{i} \neq K$ and let $f_{1} \in \mid{ }_{i=1}^{n} K e r D_{i}, f_{1} \in R \backslash K$. Then there exists a transcendence basis $\left\{f_{1}, K, f_{n}\right\}$ of $R$ over $K$ and the subfield $K\left(f_{1}, K, f_{n}\right)$ is isomorphic to the field $K\left(x_{1}, K, x_{n}\right)$. The function $f_{1}$ defines the derivation $S$ of the field $K\left(f_{1}, K, f_{n}\right)$ and this derivation can be uniquely extended to the derivation $S$ of $K\left(x_{1}, K, x_{n}\right)$ (we keep the same notation for the extended derivation). But $S=\sum_{i=1}^{n} s_{i} D_{i}$ for some $s_{i} \in R$ and therefore $S\left(f_{1}\right)=\sum_{i=1}^{n} S_{i} D_{i}\left(f_{1}\right)=0$ by the choice of the element $f_{1}$. This is impossible because $S\left(f_{1}\right)=1$. The obtained contradiction shows that $\mathrm{I}_{\mathrm{i}=\mathrm{I}}^{\mathrm{n}} \mathrm{KerD}_{\mathrm{i}}=\mathrm{K}$

Corollary 1 If $L$ is an abelian subalgebra of $\mathbb{H}_{3}(K)$ and $r_{R} L=n$, then $\operatorname{dim}_{\mathrm{K}} \mathrm{L}=\mathrm{n}$.

Proof. Let $D_{1}, K, D_{n}$ be a basis of $L$ over $R$. Then any element $D \in L$ is of the form $D=\sum_{i=1}^{n} s_{i} D_{i}$ for some $s_{i} \in R$. Since $\left[D_{i}, D\right]=0=\sum_{j=1}^{n} D_{i}\left(s_{j}\right) D_{j}$ we have that $D_{i}\left(s_{j}\right)=0, i, j=1, K, n$. By Lemma $3, s_{i} \in K$ and $D_{1}, K, D_{n}$ is a basis of $L$ over $K$. Thus $\operatorname{dim}_{K} L=n$.

Theorem 1 Let $L$ be a solvable subalgebra of the Lie algebra $W_{3}(K)$. If $L$ has an abelian ideal $I$ of rank 3 over $R$, then $L$ is isomorphic to a solvable subalgebra of the general affine Lie algebra aff $f_{3}(K)$. In particular $3 \leq \operatorname{dim}_{K} L \leq 9$.

Proof. Take any basis $D_{1}, D_{2}, D_{3}$ of the ideal I over the field $R$. Then any element $D \in L$ can be written in the form

$$
D=s_{1} D_{1}+s_{2} D_{2}+s_{3} D_{3}, \quad s_{i} \in R \text {. }
$$


Since $\left[D_{i}, D\right]=D_{i}\left(s_{1}\right) D_{1}+D_{i}\left(s_{2}\right) D_{2}+D_{i}\left(s_{3}\right) D_{3} \in I$ we have by Lemma 4 that $D_{i}\left(s_{j}\right) \in K, i, j=1,2,3$. So we can correspond to any element $D \in L$ the matrix

$$
B_{D}=\left(\begin{array}{lll}
D_{1}\left(s_{1}\right) & D_{1}\left(s_{2}\right) & D_{1}\left(s_{3}\right) \\
D_{2}\left(s_{1}\right) & D_{2}\left(s_{2}\right) & D_{2}\left(s_{3}\right) \\
D_{3}\left(s_{1}\right) & D_{3}\left(s_{2}\right) & D_{3}\left(s_{3}\right)
\end{array}\right) \in M_{3}(K)
$$

Denote by $S$ the set of all columns of such matrices $B_{D}$, where $D$ runs over the subalgebra $\mathrm{L}$. Since $\mathrm{S} \subseteq \mathrm{K}^{3}$, the three-dimension vector space over $\mathrm{K}$, we have $d=r k_{K} S \leq 3$. If $d=0$, then all columns for all $D \in L$ are zero and therefore $s_{i} \in K, i=1,2,3$ by Lemma 3. This means $L=1$. So we can assume that $d \geq 1$.

Case 1. $d=1$. Then there exists an element $D \in L \backslash I$ which can be written in the form $D=S_{1} D_{1}+S_{2} D_{2}+S_{3} D_{3}$ such that all columns of $S$ are proportional to the column $\left(D_{1}\left(S_{1}\right), D_{2}\left(S_{1}\right), D_{3}\left(S_{1}\right)\right)^{\top}$ (here $\cdot^{\top}$ denotes the transpose of the row) of the corresponding matrix $B_{D}$. Take any element $\left(D_{1}(t), D_{2}(t), D_{3}(t)\right)^{\top} \in S$. Then there exists $\gamma \in K$ such that

$$
\left(D_{1}(t), D_{2}(t), D_{3}(t)\right)^{\top}=\gamma\left(D_{1}\left(s_{1}\right), D_{2}\left(s_{1}\right), D_{3}\left(s_{1}\right)\right)^{\top} \text {. }
$$

It follows from the last equality that

$$
D_{1}\left(t-\gamma s_{1}\right)=D_{2}\left(t-\gamma s_{1}\right)=D_{3}\left(t-\gamma s_{1}\right)=0
$$

By Lemma 3 we obtain $t-\gamma s_{1}=\delta$ for some $\delta \in K$, i.e. $t=\gamma s_{1}+\delta$. The latter means that for any element $D \in L, \quad D=t_{1} D_{1}+t_{2} D_{2}+t_{3} D_{3}, t_{i} \in R$ the corresponding matrix $B_{D}$ has the columns $\left(D_{1}\left(t_{i}\right), D_{2}\left(t_{i}\right), D_{3}\left(t_{i}\right)\right)^{\top} i=1,2,3$ with $t_{i}=f_{i}(s)$, deg $f_{i} \leq 1, f_{i} \in K[t]$. Since $\left(D_{1}\left(s_{1}\right), D_{2}\left(s_{1}\right), D_{3}\left(s_{1}\right)\right)^{\top}$ is nonzero we can assume without loss of generality that $D_{1}\left(s_{1}\right)=1, D_{2}\left(s_{1}\right)=\gamma_{2}, D_{3}\left(s_{1}\right)=\gamma_{3}$ for some $\gamma_{2}, \gamma_{3} \in \mathrm{K}$. Put

$$
D_{1^{\prime}}=D_{1}, D_{2}=D_{2}-\gamma_{2} D_{1}, D_{3}=D_{3}-\gamma_{3} D_{1} \text {. }
$$

Then $D_{1^{\prime}}\left(S_{1}\right)=1, D_{2^{\prime}}\left(S_{1}\right)=0, D_{3^{\prime}}\left(S_{1}\right)=0$ and $D_{1^{\prime}}, D_{2^{\prime}}, D_{3^{\prime}}$ form a basis of 1 over $R$. Let $D=t_{1} D_{1}+t_{2} D_{2}+t_{3} D_{3}$ be an arbitrary element in $L$ and $t_{i}=\gamma_{i} s_{i}+\delta_{i}, i=1,2,3$. Then the map $\varphi: L \rightarrow$ aff $_{3}(K)$ which is defined by the rule: $\varphi\left(D_{i}\right)=x_{i}, \varphi\left(s_{1} D_{i}\right)=x_{1} x_{i}$ and further by linearity, is an embedding of $L$ into the Lie algebra aff $f_{3}(K)$.

Case 2. $d=r k_{k} S=2$. Then there exist linearly independent columns on the set $\mathrm{S}$ of the form

$$
\left(D_{1}\left(s_{1}\right), D_{2}\left(s_{1}\right), D_{3}\left(s_{1}\right)\right)^{\top},\left(D_{1}\left(s_{2}\right), D_{2}\left(s_{2}\right), D_{3}\left(s_{2}\right)\right)^{\top}
$$

(these columns can belong to different matrices $B_{D}, D \in L$ ). Therefore any column $\left(D_{1}(t), D_{2}(t), D_{3}(t)\right)^{\top} \in S$ is a linear combination of columns in (2). One can easily show that $t=f\left(s_{1}, s_{2}\right)$ for some polynomial $f \in K[u, v]$, deg $f \leq 1$. Note that the rank of the matrix

$$
\left(\begin{array}{ll}
D_{1}\left(s_{1}\right) & D_{1}\left(s_{2}\right) \\
D_{2}\left(s_{1}\right) & D_{2}\left(s_{2}\right) \\
D_{3}\left(s_{1}\right) & D_{3}\left(s_{2}\right)
\end{array}\right)
$$


is equal to 2. Without loss of generality one can assume that the first and second rows of this matrix are linearly independent. But then there exist $\gamma_{1}, \gamma_{2} \in \mathrm{K}$ such that

$$
(1,0)=\gamma_{1}\left(D_{1}\left(s_{1}\right), D_{1}\left(s_{2}\right)\right)+\gamma_{2}\left(D_{2}\left(s_{1}\right), D_{2}\left(s_{2}\right)\right) \text {. }
$$

Denoting $D_{1^{\prime}}=\gamma_{1} D_{1}+\gamma_{2} D_{2}$ we have $D_{1^{\prime}}\left(S_{1}\right)=1, D_{1^{\prime}}\left(s_{2}\right)=0$. Analogously one can find $\delta_{1}, \delta_{2} \in \mathrm{K}$ such that the element $\mathrm{D}_{2^{\prime}}=\delta_{1} \mathrm{D}_{1}+\delta_{2} \mathrm{D}_{2}$ has properties $D_{2}\left(s_{1}\right)=0, D_{2^{\prime}}\left(s_{2}\right)=1$.

Further, the third row of the matrix (3) is a linear combination of the first and second rows and therefore $\left(D_{3}-\mu_{1} D_{1}-\mu_{2} D_{2}\right)\left(s_{i}\right)=0, i=1,2$. Denoting $D_{3}=D_{3}-\mu_{1} D_{1}-\mu_{2} D_{2}$ we obtain $D_{i^{\prime}}\left(s_{j}\right)=\delta_{i j}, i=1,2,3, j=1,2$. If $D \in L$ is an arbitrary element, then $D=t_{1} D_{1}+t_{2} D_{2}+t_{3} D_{3}$ for some $t_{1}, t_{2}, t_{3} \in R$. Since $t_{i}=f_{i}\left(s_{1}, s_{2}\right)$, deg $f_{i} \leq 1$ we see that $L$ can be embedded in the Lie algebra $\operatorname{aff}_{3}(K)$.

Case $3 \mathrm{rk}_{\mathrm{K}} \mathrm{S}=3$ can be considered analogously.

Subalgebras with abelian ideals of $r k \leq 2$ over $R$.

Lemma 4 Let $L$ be a subalgebra of the Lie algebra $\mathbb{H}_{n}(K)$ and I be an ideal of $L$. If $F=F(I)$ is the field of constants for $I$ in $R$, then $D(F) \subseteq F$ for any element $D \in L$.

Proof. Let $D \in L$ and $r \in F$ be arbitrarily chosen. Then for any $D_{1} \in I$ we have $D_{1}(r)=0$ and therefore

$$
0=D\left(D_{1}(r)\right)=D_{1}(D(r))+\left[D, D_{1}\right](r) \text {. }
$$

Since $\left[D, D_{1}\right] \in I$ we have $\left[D, D_{1}\right](r)=0$ and consequently $D_{1}(D(r))=0$. The latter means that $D(r) \in F$ because the element $D_{1}$ was arbitrarily chosen in the ideal $\mathrm{I}$. Thus $\mathrm{D}(\mathrm{F}) \subseteq \mathrm{F}$.

Theorem 2 Let $L$ be a solvable finite dimensional subalgebra of the Lie algebra $W_{3}(K)$ with $r k_{R} L=3$. If $L$ has an ideal $I$ of rank 2 over $R$ and $F=F(L)$ is the field of constants of $I$ in $R$, then the Lie algebra $L$ is contained in the subalgebra $\bar{L}=F \bar{I}+L$ of $H_{3}(K)$ where $\bar{I}=(R I) \cap L$. The Lie algebra $\bar{L}$ is solvable, $F \bar{I}$ is its ideal of rank 2 over $R$ which is isomorphic to a subalgebra of aff $_{2}(F)$. The Lie algebra $\bar{L}$ is an extension of the ideal $F \bar{I}$ by a Lie algebra of dimension 1 or 2 over $\mathrm{K}$.

Proof. The intersection $\bar{I}=(R I) \cap L$ is an ideal of the Lie algebra $L$ with $r k_{R} \bar{L}=2$ and $\operatorname{dim}_{K} L / \bar{T} \leq 2$ (see [8]). Let $F$ be the field of constants for $\mathrm{I}$ in $R$. Since $D(F) \subseteq F$ for any $D \in L$ (by Lemma 4), the subalgebra $F \bar{I}$ of the algebra $\mathbb{H}_{3}(K)$ is an ideal of the Lie algebra $F \bar{I}+L$. One can easily show that $r k_{R} \bar{I}=2$. By Theorem 1 of the paper [6], the Lie algebra Fì (as a Lie algebra over the field $F)$ is isomorphic to a subalgebra of the Lie algebra aff $f_{2}(F)$. Since $\operatorname{dim}_{K} L / \bar{I} \leq 2$, it holds obviously $\operatorname{dim}_{K} L+F \bar{I} / F \bar{I} \leq 2$. Note that the Lie algebra $\mathrm{L}+\mathrm{F} \overline{\mathrm{I}}$ is in general case of infinite dimension over $K$ although $\operatorname{dim}_{F} F \bar{I} \leq 7$ (the sum $F \bar{I}+L$ is not in general a Lie algebra over $F$ but only over the field $\mathrm{K}$ ).The proof is complete.

Further notations are taken from Theorem 2. Let $I_{1}=K D_{1}$ be a onedimensional ideal of $L$ lying in $I$ and $K D_{2}+I_{1}$ be an ideal of the quotient 
algebra $L / I_{1}$ lying in $I / I_{1}$ (such ideals do exist because $L$ is solvable and $K$ is algebraically closed). Let $K D_{3}+\bar{l}$ be one-dimensional ideal of the Lie algebra $L / \bar{l}$. Then $D_{1}, D_{2}, D_{3}$ are linearly independent over $R$ and form $a$ basis of $R L$ over $R$. By the choice of $D_{1}$ and $D_{2}$ there exist $\lambda_{1}, \lambda_{2} \in K$ and $g_{2} \in F$ such that

$$
\left[D_{3}, D_{1}\right]=\lambda_{1} D_{1},\left[D_{3}, D_{2}\right]=\lambda_{2} D_{2}+g_{2} D_{1} \text {. }
$$

The next statement gives more detailed description of the Lie algebra $\overline{\mathrm{L}}=\mathrm{F} \overline{\mathrm{I}}+\mathrm{L}$.

Proposition 1 Let $L \subseteq W_{3}(K)$ be a solvable finite dimensional subalgebra of rank 3 over $R$ with dimL $>6$. Under conditions of Theorem 2 either there exist $r_{1}, r_{2} \in R$ with $D_{i}\left(r_{j}\right)=\delta_{i j}, i, j=1,2$ and every element $D \in F \bar{I}$ is of the form $D=f_{1}\left(r_{1}, r_{2}\right) D_{1}+f_{2}\left(r_{1}, r_{2}\right) D_{2}, f_{i} \in K\left[t_{1}, t_{2}\right]$, deg $f_{i} \leq 1$ or there exists $r_{i} \in R$, $\mathrm{i}=1$ or $\mathrm{i}=2$ with $\mathrm{D}_{\mathrm{i}}\left(r_{\mathrm{j}}\right)=\delta_{i j}$ and every element $\mathrm{D} \in \mathrm{F} \bar{I}^{-}$is of the form $D=g_{1}\left(r_{i}\right) D_{1}+g_{2}\left(r_{i}\right) D_{2}$, deg $g_{j} \leq 1$. Then $D_{3}\left(r_{1}\right)=-\lambda_{1} r_{1}-g_{2} r_{2}, D_{3}\left(r_{2}\right)=-\lambda_{2} r_{2}$. If $\operatorname{dim}_{K} L / \bar{T}=2$, then there exists $\bar{D} \in L \backslash\left(K D_{3}+\bar{l}\right)$ such that $\bar{D}=r_{3} D_{3}+s_{2} D_{2}$, $r_{3} \in R, D_{3}\left(r_{3}\right)=1, D_{1}\left(r_{3}\right)=D_{2}\left(r_{3}\right)=0, D_{1}\left(s_{2}\right)=0$, and in this case $\lambda_{1}=0, g_{2}=0$, $s_{2}=\lambda_{2} r_{2} r_{3}+f, f \in K$.

Proof. Repeating considerations from the proof of Theorem 1 one can find either elements $r_{1}, r_{2}$ with $D_{i}\left(r_{j}\right)=\delta_{i j}, i, j=1,2$ or an element $r \in R$ such that either $D_{1}(r)=1, D_{2}(r)=\gamma$ or $D_{1}(r)=\delta, D_{2}(r)=1$ using only transformations of columns of the matrix $B_{D}=\left(\begin{array}{ll}D_{1}\left(s_{1}\right) & D_{1}\left(s_{2}\right) \\ D_{2}\left(s_{1}\right) & D_{2}\left(s_{2}\right)\end{array}\right)$. If $\delta \neq 0$ we can consider elements $D_{2}=D_{2}-\delta D_{1}, D_{1^{\prime}}=D_{1}$, and in this case $D_{1^{\prime}}(r)=0, D_{z^{\prime}}(r)=1$. So we can assume that either $D_{1}(r)=1, D_{2}(r)=0$ or $D_{1}(r)=0, D_{2}(r)=1$ and $r$ is either $r_{1}$ or $r_{2}$.

Let us consider the action of elements $D_{i}$ on $r_{i}, s_{j}, i=1,2,3, j=2,3$.

Since $D_{1}\left(r_{1}\right)=1$ we have $D_{3}\left(D_{1}\left(r_{1}\right)\right)=0$ and therefore

$$
D_{1}\left(D_{3}\left(r_{1}\right)\right)=D_{3}\left(D_{1}\left(r_{1}\right)\right)-\left[D_{3}, D_{1}\right]\left(r_{1}\right)=0-\lambda_{1} D_{1}\left(r_{1}\right)=-\lambda_{1} \text {. }
$$

It follows from the equalities $D_{1}\left(D_{3}\left(r_{1}\right)\right)=-\lambda_{1}$ and $D_{1}\left(-\lambda_{1} r_{1}\right)=-\lambda_{1}$ that $D_{1}\left(D_{3}\left(r_{1}\right)+\lambda_{1} r_{1}\right)=0$, i.e. $D_{3}\left(r_{1}\right)=-\lambda_{1} r_{1}+s^{\prime}$ for some $s^{\prime} \in \operatorname{Ker} D_{1}$. A nalogously the equality

$$
D_{2}\left(d_{3}\left(r_{1}\right)\right)=D_{3}\left(D_{2}\left(r_{1}\right)\right)-\left[D_{3}, D_{2}\right]\left(r_{1}\right)
$$

implies $D_{3}\left(r_{1}\right)=-g_{2} r_{2}+s^{\prime \prime}$ for some $s^{\prime \prime} \in \operatorname{Ker} D_{2}$. Applying $D_{1}$ to both sides of the obtained equality $-\lambda_{1} r_{1}+s^{\prime}=-g_{2} r_{2}+s^{\prime \prime}$ we get $-\lambda_{1}=D_{1}\left(s^{\prime \prime}\right)$. After applying $D_{2}$ to the same equality we get $D_{2}\left(s^{\prime}\right)=-g_{2}$. But then $s^{\prime \prime}+\lambda_{1} r_{1} \in \operatorname{Ker} D_{1}$. Since $s^{\prime \prime}+\lambda_{1} r_{1} \in \operatorname{KerD}_{2} \quad$ we have $s^{\prime \prime}+\lambda_{1} r_{1} \in \operatorname{Ker}_{1} \cap \operatorname{Ker}_{2}=F$. Thus $s^{\prime \prime}=-\lambda_{1} r_{1}+v_{1}$ for some $v_{1} \in F$ It follows from the equality $-\lambda_{1} r_{1}+s^{\prime}=-g_{2}-\lambda_{1} r_{1}+v_{1}$ that $s^{\prime}=-g_{2} r_{2}+v_{1}$. Finally we get $D_{3}\left(r_{1}\right)=-\lambda_{1} r_{1}-g_{2} r_{2}+v_{1}, v_{1} \in F$.

Analogously it follows from the equalities

$$
D_{2}\left(D_{3}\left(r_{2}\right)\right)=D_{3}\left(D_{2}\left(r_{2}\right)\right)-\left[D_{3}, D_{2}\right]\left(r_{2}\right)=0-\left(\lambda_{2} D_{2}+g_{2} D_{1}\right)\left(r_{2}\right)=-\lambda_{2}
$$

that $D_{3}\left(r_{2}\right)=-\lambda_{2} r_{2}+t^{\prime}$ for some $t^{\prime} \in$ KerD $D_{2}$ and finally 


$$
D_{3}\left(r_{2}\right)=-\lambda_{2} r_{2}+v_{2}, v_{2} \in F
$$

Without loss of generality we can change $D_{3}$ by $D_{3}=D_{3}-v_{1} D_{1}-v_{2} D_{2}$. Then $D_{3^{\prime}}\left(r_{1}\right)=-\lambda_{1} r_{1}-g_{2} r_{2}, D_{3}\left(r_{2}\right)=-\lambda_{2} r_{2}$. Returning to the old notation we have $D_{3}\left(r_{1}\right)=-\lambda_{1} r_{1}-g_{2} r_{2}, D_{3}\left(r_{2}\right)=-\lambda_{2} r_{2}$.

Let now $\operatorname{dim}_{K} L / \bar{T}=2$ and $\bar{D}=r_{3} D_{3}+s_{1} D_{1}+s_{2} D_{2}$ be any element of $L \backslash\left(K D_{3}+I\right)$. Then

$$
\begin{aligned}
{\left[\bar{D}, D_{3}\right] } & =\left[r_{3} D_{3}+s_{1} D_{1}+s_{2} D_{2}, D_{3}\right]= \\
& =-D_{3}\left(r_{3}\right) D_{3}-D_{3}\left(s_{1}\right) D_{1}-s_{1}\left[D_{1}, D_{3}\right]-D_{3}\left(s_{2}\right) D_{2}-s_{2}\left[D_{2}, D_{3}\right]= \\
& =-D_{3}\left(r_{3}\right) D_{3}+\left(-D_{3}\left(s_{1}\right)+\lambda_{1} s_{1}+s_{2} g_{2}\right) D_{1}+\left(-D_{3}\left(s_{2}\right)+\lambda_{2} s_{2}\right) D_{2} .
\end{aligned}
$$

It follows from these equalities that $D_{3}\left(r_{3}\right)=-\gamma$, where $\gamma$ is taken from the equality $\left[\bar{D}, D_{3}\right]=\gamma D_{3}+\mathbb{D}$, where $\boldsymbol{Q} \in \overline{\mathrm{I}}$. A nalogously the equality

$$
\left[r_{3} D_{3}+s_{1} D_{1}+s_{2} D_{2}, D_{1}\right]=\mu D_{1}
$$

for some $\mu \in K$ implies $D_{1}\left(r_{3}\right)=0, D_{1}\left(s_{2}\right)=0$. The equality

$$
\left[r_{3} D_{3}+s_{1} D_{1}+s_{2} D_{2}, D_{2}\right]=f_{1} D_{1}+f_{2} D_{2}
$$

for some $f_{1}, f_{2} \in F$ yields $D_{3}\left(r_{3}\right)=0$. Summarizing we get

$$
D_{1}\left(r_{3}\right)=D_{2}\left(r_{3}\right)=0, \quad D_{3}\left(r_{3}\right)=1, \quad D_{1}\left(s_{2}\right)=0 \text {. }
$$

Since $\left[\bar{D}, D_{1}\right]=\theta D_{1}$ for some $\theta \in K$ we have

$$
\left[r_{3} D_{3}+s_{1} D_{1}+s_{2} D_{2}, D_{3}\right]=\left(\lambda_{1} r_{3}-D_{1}\left(s_{1}\right)\right) D_{1}
$$

and therefore $\lambda_{1} r_{3}-D_{1}\left(s_{1}\right)=\theta$. Thus $D_{1}\left(s_{1}\right)=\lambda_{1} r_{3}+\theta, \theta \in K$. Further $\left[\bar{D}, D_{2}\right]=f_{1} D_{1}+f_{2} D_{2}$ for some $f_{1}, f_{2} \in F$. A nalogously $\left[r_{3} D_{3}+s_{1} D_{1}+s_{2} D_{2}, D_{2}\right]=$ $=\left(r_{3} g_{2}-D_{2}\left(s_{1}\right)\right) D_{1}+\left(\lambda_{2} r_{2}-D_{2}\left(s_{2}\right)\right) D_{2}$ and therefore

$$
D_{2}\left(s_{1}\right)=g_{2} r_{3}-f_{2}, \quad D_{2}\left(s_{2}\right)=\lambda_{2} r_{3}-f_{2} \text {. }
$$

But we have

$$
s_{1}=g_{2} r_{2} r_{3}-r_{2} f_{2}+f_{3}, s_{2}=\lambda_{2} r_{2} r_{3}-r_{2} f_{2}+f_{4}
$$

for some $f_{3}, f_{4} \in F$. It was proved early that $D_{1}\left(s_{1}\right)=\lambda_{1} r_{3}+\theta, \theta \in K$, so we have $s_{1}=\lambda_{1} r_{1} r_{3}+\theta r_{1}+f_{5}$ for some $f_{5} \in F$. Applying $D_{2}$ to the both sides of the equality

$$
\lambda_{1} r_{1} r_{3}+\theta r_{1}+f_{5}=g_{2} r_{2} r_{3}-r_{2} f_{2}+f_{3}
$$

we get $g_{2} r_{3}-f_{2}=0$. But $r_{1}, r_{2}, r_{3}$ are linearly independent over $F$, so the last equality yields $g_{2}=0$. The equality (7) is now of the form

$$
\lambda_{1} r_{1} r_{3}+\theta r_{1}+f_{5}=-r_{2} f_{2}+f_{3}
$$

Applying $D_{2}$ to the both sides of this equality we get $f_{2}=0$. Therefore $\lambda_{1} r_{1} r_{3}+\theta r_{1}+f_{5}=f_{3}$. A pplying $D_{1}$ to the both sides of the last equality we get $\lambda_{1} r_{3}+\theta=0$. Since $r_{3} \notin K$ we have $\lambda_{1}=0$ and therefore $s_{1}=0$. A nalogously we can assume that $f_{4}=0$ and $s_{2}=\lambda_{2} r_{2} r_{3}$. So we have

$$
s_{1}=0, s_{2}=\lambda_{2} r_{2} r_{3}, g_{2}=0, f_{2}=0, \lambda_{1}=0 \text {. }
$$

These equalities means that

$$
\left[D_{3}, D_{1}\right]=0,\left[D_{3}, D_{2}\right]=\lambda_{2} D_{2}, \bar{D}=r_{3} D_{3}+s_{2} D_{2} \text {, }
$$

where $s_{2}=\lambda_{2} r_{2} r_{3}, D_{i}\left(r_{j}\right)=\delta_{i j}, i, j=1,2,3$. The proof is complete. 
1. Amaldi U. Contributo all determinazione dei gruppi continui finiti dello spazio ordinario I // Giornale Mat. Battaglini Prog. Studi Univ. Ital. - 1901. - 39. P. 273- 316.

2. Amaldi U. Contributo all determinazione dei gruppi continui finiti dello spazio ordinario II // Giornale Mat. Battaglini Prog. Studi Univ. Ital. - 1902. - 40. P. 105- 141.

3. Bondarenko V. M., Petravchuk. A. P. Wildness of the problem of classifying nilpotent Lie algebras of vector fields in four variables // arXiv: 18.03.09772v1 [math. RA].

4. Gonzalez-Lopez A., Kamran N. and Olver P. J. Lie algebras of differential ope rators in two complex variables // Amer. J . Math. - 1992. - 114. - P. 1163- 1185.

5. Gonzalez-Lopez A., Kamran N. and Olver P. J. Lie algebras of vector fields in the real plane // Proc. London Math. Soc. - 1992. - 64(3), - № 2. - P. 339- 368.

6. Klimenko I., Lysenko S., Petravchuk A. Lie algebras of derivations with abelian ideals of maximal rank // Scientific Bulletin of Uzhhorod University, Ser. Mathematics and Informatics. - 2017. - 31. - P. 75- 81 (in Ukrainian).

7. Lie S. Theorie der Transformationsgruppen. - Leipzig, 1888, 1890, 1893. Vol. 1- 3.

8. Ie. O. Makedonskyi and Petravchuk A. P. On nilpotent and solvable Lie algebras of derivations // J . of Algebra. - 2014. - 401. - P. 245- 257

9. Nowicki A. Polynomial Derivations and their Rings of Constants. - Torun: Uniwersytet Mikolaja Kopernika, 1994. - 176 p.

10. Petravchuk A.P. On nilpotent Lie algebras of derivations of fraction fields // Algebra and Discrete Math. - 2016. - 22. - P. 118- 131

\section{РОЗВ'ЯЗНІ АЛГЕБРИ ЛІ ДИФЕРЕНЦІЮВАНЬ КІЛЕЦЬ МНОГОЧЛЕНІВ ВІД ТРЬОХ ЗМІННИХ}

Нехай $\mathrm{K}$ - алгебраїчно замкнене поле характеристики нуль, $\mathrm{A}=\mathrm{K}\left[\mathrm{x}_{1}, \mathrm{x}_{2}, \mathrm{X}_{3}\right]$ кільие многочленів від тръох змінних $i \mathrm{R}=\mathrm{K}\left(\mathrm{X}_{1}, \mathrm{X}_{2}, \mathrm{X}_{3}\right)$ - поле рачіональних функиій. Якщо $\mathrm{L}$ - підалгебра алгебри лі $\mathrm{W}_{3}(\mathrm{~K})$ всіх $\mathrm{K}$-дифберенціювань кільия $\mathrm{A}$, то $\mathrm{RL} \epsilon$ алгеброю Лі над $\mathrm{K} i \operatorname{dim}_{\mathrm{R}} \mathrm{RL}$ називається рангом алгебри $\mathrm{L}$ над $\mathrm{R}$. Вивчаються підалгебри $\mathrm{L}$ рангу 3 над $\mathrm{R}$ алгебри Лі $\mathrm{W}_{3}(\mathrm{~K})$. Доведено, щуо якщо $\mathrm{L}$ містить абелевий ідеал I рангу 3 над $\mathrm{R}$, то L ізоморбна підалгебрі загальної афбінної алгебри Лi aff $_{3}(\mathrm{~K})$. Якщо $\mathrm{L}$ має iдеал $\mathrm{I}_{3} \mathrm{rk}_{\mathrm{R}} \mathrm{I}=2$, то $\mathrm{L}$ міститься в підалгебрі $\overline{\mathrm{L}}$ алгебри $\mathrm{W} / \mathrm{K}(\mathrm{K})=\operatorname{Der}_{\mathrm{K}} \mathrm{R}$, де $\mathrm{L}$ - розширення делкої підалгебри із $\mathrm{aff}_{2}(\mathrm{~F})$ за допомогою підалгебри розлірності $\leq 2$, а $\mathrm{F}-$ поле констант для $\mathrm{I}$ в $\mathrm{R}$.

\section{РАЗРЕШИМЫЕ АЛГЕБРЫ ЛИ ДИФФЕРЕНЦИРОВАНИЙ КОЛЕЦ МНОГОЧЛЕНОВ ОТ TPEX ПЕРЕMEHНЫХ}

Пусть $\mathrm{K}$ - алгебраически залкнутое поле характеристики нуль, $\mathrm{A}=\mathrm{K}\left[\mathrm{x}_{1}, \mathrm{x}_{2}, \mathrm{x}_{3}\right]$ - кольио многочленов от трех переменных и $\mathrm{R}=\mathrm{K}\left(\mathrm{x}_{1}, \mathrm{x}_{2}, \mathrm{X}_{3}\right)$ - поле рациональных фбункций. Если L-подалгебра алгебры Ли $\mathrm{W}_{3}(\mathrm{~K})$ всех $\mathrm{K}$-диферениирований кольияа $\mathrm{A}$, то $\mathrm{RL}$ является алгеброй Ли над $\mathrm{K}$ u $\operatorname{dim}_{\mathrm{R}} \mathrm{RL}$ називается рангом алгебры $\mathrm{L}$ над $\mathrm{R}$. Исследуются підалгебры $\mathrm{L}$ ранга 3 над $\mathrm{R}$ алгебры Ли $\mathrm{W}_{3}(\mathrm{~K})$. Доказано, что если L содержит абелев идеал I ранга 3 над R, то L изоморбна подалгебре общей афинной алгебры Ли $\mathrm{aff}_{3}(\mathrm{~K})$. Если $\mathrm{L}$ содержит идеал I c $\mathrm{rk}_{\mathrm{R}} \mathrm{I}=2$, то $\mathrm{L}$ содержится в подалгебре $\mathrm{L}$ алгебры $\mathrm{V}_{3}(\mathrm{~K})=\operatorname{Der}_{\mathrm{K}} \mathrm{R}$, где $\mathrm{L}$ расширение некоторой подалгебры из $\mathrm{aff}_{2}(\mathrm{~F})$ с помощъю подалгебры размерности $\leq 2$, а $\mathrm{F}$ - поле констант для І в $\mathrm{R}$.

Київ. нац. ун-т 